\title{
Do clinical trials meet current care needs? Views of digestive oncology specialists in Galicia (Spain) using the Delphi method
}

\section{Ana Fernández Montes}

Complejo Hospitalario de Ourense

Nieves Martínez Lago

Complexo Hospitalario Universitario A Coruña

Juan de la Cámara Gomez

Complexo Hospitalario Universitario de Ferrol (Ferrol)

Elena María Brozos Vázquez

Complexo Hospitalario Universitario de Santiago de Compostela (Santiago de Compostela)

\section{Sonia Candamio Folgar}

Complexo Hospitalario Universitario de Santiago de Compostela (Santiago de Compostela)

\section{Marta Carmona Campos}

Hospital Universitario Lucus Augusti

\section{Antía Cousillas Castiñeiras}

Complejo Hospitalario de Pontevedra

\section{Marta Covela Rúa}

Hospital Universitario Lucus Augusti

Elena Gallardo Martín

Complejo Hospitalario de Pontevedra

\section{Paula González Villarroel}

Hospital Álvaro Cunqueiro (Vigo)

\section{Begoña Graña Suarez}

Complexo Hospitalario Universitario A Coruña

\section{Mónica Jorge Fernández}

Hospital Álvaro Cunqueiro (Vigo)

\section{María Luz Pellón Augusto}

Complexo Hospitalario Universitario de Ferrol (Ferrol)

\section{Guillermo Quintero Aldana}

Hospital Universitario Lucus Augusti

\section{Carlos Romero Reinoso}

Povisa Hospital

\section{Mercedes Salgado Fernández}


Complejo Hospitalario de Ourense

Francisca Vázquez Rivera

Complexo Hospitalario Universitario de Santiago de Compostela (Santiago de Compostela)

Ana Ayuso_Álvarez

Instituto de Salud Carlos III

José Carlos Méndez Méndez

Sanatorio Nosa Señora dos Ollos Grandes (Lugo).

Dante R Culqui ( $\sim$ danteroger@hotmail.com )

Sociosanitario Isabel Roig (Barcelona)

\section{Righ sens Working group}

Complejo Hospitalario de Ourense

\section{Research Article}

Keywords: Views, oncology specialists, Delphi method

Posted Date: March 12th, 2021

DOl: https://doi.org/10.21203/rs.3.rs-228852/v1

License: (1) (1) This work is licensed under a Creative Commons Attribution 4.0 International License. Read Full License 


\section{Abstract}

Background: In recent years, abundant scientific evidence has been generated based on clinical trials (CT) in the field of oncology. In spite of this, there are still major disagreements among the group when it comes to establishing a treatment and therapies for these kinds of patients. The general objective of this paper is to find out the extent to which decision-making is based on knowledge of the most recent CT. Its specific objectives are to pinpoint difficulties with decision-making based on the CT performed and find out the motivations patients and clinicians have when taking part in a CT.

Methodology: Combined, prospective study, based on the Delphi method.

Results: A lack of correspondence between the people who take part in CT and patients who come for consultation has been identified. Healthcare professionals' motivations for taking part in a CT include finding better treatment for the patient and boosting their research career. A need for training in analysing and interpreting CT has also been identified and a lack of trust in the results of CT financed by the pharmaceutical industry itself has been perceived.

Conclusion: There is a difficulty in selecting oncological treatment due to the lack of correspondence between the patients included in the CT and patients seen in consultation. In this process, real world data studies may be highly useful, as may be providing this group with greater training in interpreting CT and their results. Aiding the professional progression of their research career is an incentive for clinicians to participate in a CT.

\section{Introduction}

Over the last decade, many publications have questioned the usefulness of CT. John P. A. loannidis raises the need to take an appropriate clinical approach to them and consider their usefulness when the results bring about a change in therapeutic decision-making ${ }^{1}$.

By 2014, approximately one million CT and tens of thousands of systematic reviews had been published, the majority of which were not clinically useful. It is estimated that $85 \%$ of the billions invested in research each year goes into $\mathrm{CT}^{2}$.

The majority of CT are designed with the aim of demonstrating the efficacy and safety of medications in order for the pharmaceutical industry to obtain medium/long-term regulatory approvals (clinical developments from early phases to phase III or final, pivotal or registration phases).

Many authors deem that if a CT is very flexible in its design, definitions or analysis, its results may be less rigorous ${ }^{3}$.

In order to have better control during the design and performance of a CT, tools have been created such as that produced by Cochrane, which seeks to set out minimum criteria to determine the quality of the 
articles analysed, and both the internal and external validity of $\mathrm{CT}^{4}$ is assessed in order to do this. In oncology there are constant therapeutic advances and, in a matter of just a few years, there has been a shift from cytotoxic drugs to monoclonal antibodies as specific therapeutic targets or immune response modulating drugs to treat a tumour. In addition, in some cases there are different combinations of all of these, which makes them more complicated to analyse.

In this speciality, there are major advances in clinical treatment for multiple types of tumour with different histologies, specific biomarkers, drug combinations and mechanisms of action. Generally speaking, precision medicine has allowed a great advance in the efficacy and safety of the various therapeutic strategies. There is thus a great amount of information concerning new drugs tested in populations that do not adequately represent the type of patients actually seen by specialists in their day-to-day clinical practice. On many occasions, this means that the results of CT are extended to special populations that are not included in them.

This problem raises questions concerning the methodology of CT. Just how prepared are we to deal with the new methodological designs necessary to analyse new treatments in all populations? Do CT represent the population we want to treat? Researchers themselves are aware of these aspects, but how do they perceive and tackle problems with CT in day-to-day clinical practice?

The RIGhT-sens study (Delivering a Right and Individualized Digestive Tumor Treatment) is intended to show the perception of medical oncology specialists who belong to the Galician Digestive Tumour Research Group (Grupo Gallego de Investigación en Tumores Digestivos de Galicia - GITuD). The aim of this group is to raise medical awareness of the influence of CT on the handling of patients with digestive tumours in Galicia and in Spain.

The general objective of this study is to find out the extent to which oncology specialists are aware of CT, both their quality and their interpretation, as well as how they use them when deciding on one treatment or another.

\section{Methodology}

\section{Type of study}

Combined (qualitative and quantitative), prospective study using the Delphi method.

The Delphi method is a systematic forecasting method, the objective of which is to obtain a consensus based on discussion by experts (oncologists) through an iterative process. It is used when there is scarce empirical evidence, the data are vague and subjective factors predominate ${ }^{5}$.

This methodology has high reliability, flexibility, dynamism and validity because it allows for anonymous participation, a heterogeneous group of experts, iteration and prolonged feedback between participants, and avoids problems of representativeness and control of the discourse by some people over others ${ }^{5}$. In 
addition, there is evidence concerning the certainty it generates in decision-making, since this responsibility is shared by all of the participants ${ }^{6}$.

\section{Design and information gathering}

A questionnaire was performed with semistructured and open questions that tackle different topics in two possible scenarios: one in which there is no $\mathrm{CT}$ in progress in the hospital and the other in which there is a CT in progress in the hospital. They were also asked about their knowledge of CT.

\section{Population and sample}

The interview questionnaire was drawn up by four experts from GITuD and filled in by another 14 oncology experts in the Galician Health Service (SERGAS) who work in eight hospitals in Galicia: Complexo Hospitalario Universitario de Orense (CHOU) (one), Complexo Hospitalario de Pontevedra (CHOP) (two), Hospital Clínico Universitario de Santiago (CHUS) (three), Complejo Hospitalario Universitario A Coruña (CHUAC) (one), Complexo Hospitalario Universitario de Ferrol (CHUF) (one), Hospital Universitario Lucus Augusti (HULA) (three), Complejo Hospitalario Universitario de Vigo (CHUVI) (two) and Hospital Povisa (one).

The survey was conducted online between 11 November and 18 December 2019.

\section{Type of analysis}

The questionnaire, made up of closed and open questions, made it possible to conduct a descriptive analysis (quantitative method) and a discourse analysis (qualitative method).

The contents were analysed in parallel by two researchers (an epidemiologist and a health anthropologist) in order to avoid intersubjectivity and triangulate the results. Once it had been collected, the information was analysed until the discourse was saturated. Consensus concerning the results and their internal consistency made it unnecessary to perform a second round of consultation.

\section{Results}

The results were obtained from analysing 30 of the 32 questions in the questionnaire, since two of them did not offer significant information (question 5, which referred to the most important weaknesses in extrapolating the results of clinical trials to the work environment, and question 26, which asked about the specialists' prior participation in clinical trials).

Following the quantitative analysis, with the results shown in table 1, and the qualitative analysis, which gathers the testimony of the experts reviewed in their cognitive, social and hospital context, the main finding observed in the study was a lack of correspondence between the people who take part in CT and the patients who come for consultation, so almost half of the oncologists (43\%) did not consider the information from them to be sufficient for therapeutic decision-making concerning their patients. 
In cases in which a CT was not being conducted in their hospital (see the testimony in table 2), the information from CT was not considered sufficient for decision-making, since the indications in the CT are individualised according to the patient to be treated (testimony 1 [T1]), and it was also stated that it is difficult to find specific evidence for some diseases or certain treatment stages (T2). Therefore, it was observed that CT are not adapted to the needs of their patients (T3), their state of health or the contextual realities in which they work $(T 4, T 5)$. So they all agreed with the need for real world data (RWD) studies as a complement to CT to assess the efficacy of treatment (T6).

Generally speaking, the majority of the experts stated that it was necessary to confirm probable diagnoses in the real population or a population with similar characteristics to those they saw in consultation.

In cases in which there was a CT in the hospital (see the testimony in table 3), more than half of the interviewees $(65 \%)$ denied receiving any kind of compensation for including patients in a CT, but if there was any financial compensation, they mentioned that the researchers do not receive it directly (T7).

With regard to the usefulness of $\mathrm{CT}$, it was stated that they were useful in clinical practice and using them as a basis offered more benefits than risks. In addition, they made it possible to find new solutions to existing problems and the fact of studying those problems in their own patients was an extra incentive (T8).

It was also detected that CT could change the paradigms of clinical practice in the short to medium term, such as when a new treatment alternative is offered (T9).

It was argued that the interest in performing a CT was normally due to patient need (T10), although on some occasions it was due to private interests.

In spite of the majority considering that the personnel were qualified to be monitors or responsible for supervising a $\mathrm{CT}$, some of them had doubts about this $(\mathrm{T} 11, \mathrm{~T} 12)$ and said that, sometimes, monitors were not sufficiently trained, due to several factors: the profile of the people performing the task (inexperienced young people) and poor working conditions, which brought about a continual change in personnel $(\mathrm{T} 13, \mathrm{~T} 14)$.

With regard to logistics for the performance of $\mathrm{CT}$, the majority mentioned that they have a data manager and a management unit in their centre $(71 \%)$, but they complained about lack of support from the hospital, in spite of the fact that it could receive benefits from performing this kind of activity (T15), and they recognised that external groups are entrusted with performing the analysis (T16, T17).

In the case of a CT being performed in a hospital, no change or improvements in the logistics or infrastructure of the hospital were perceived as a consequence of it, so it also had no effect on recruitment or inclusion of patients (T18). 
Among the factors that may influence the participation of patients in CT, they mentioned the patients' socio-economic situation (T19) and the number of tests to be performed on patients and their comorbidities (T20, T21).

With regard to the criteria for selecting patients, lack of alternatives or treatment is what motivated most of the specialists to include their patients in a CT, due to considering it to be the best option for them (T23, T24). Almost all of them had recommended a CT at some time since they were convinced that it was the most beneficial therapeutic option ( $86 \%$ ), based on the medical principle of primum non nocere $(\mathrm{T} 25, \mathrm{~T} 26)$. They stated that they did not consider themselves conditioned by financial incentives (T22).

More than half of the interviewees (64\%) commented that it is difficult to find the ideal patient to include in a CT and that they found it hard to recruit patients who met all of the criteria and, also, that they were not likely to accept.

One of the reasons they all recognised as a motivation for performing a CT was the importance of being the author of a CT, since it benefits them from the viewpoint of their CV (T27) and because being an author of a publication is form of recognition of their work and a reward for it (T28, T29), although the majority did not consider it to be an indispensable requirement (T30), instead the important thing was to offer a possible better treatment alternative to patients (T31).

More than half of them had issued a publication prior to their first CT (65\%); almost $30 \%$ of these had published during the resident stage.

One influential factor in the decision to participate in a clinical trial, according to $43 \%$ of the interviewees, was the financial incentive (T32, T33, T34), but this had a different influence depending on whether they were patients or professionals. In the case of patients, their poor economic situation was mentioned and, among health professionals, the main reason was to provide a better treatment option to the patient. Almost all of them agreed that CT are useful when they offer new alternatives to patients (93\%) and when they provide data about treatment sequences or subpopulations $(\mathrm{T} 35, \mathrm{~T} 36)$.

Regarding their knowledge of CT (see the testimonies in table 4), the majority were aware of biological markers and some research techniques such as biases, although only $57 \%$ of the specialists managed to mention selection bias and very few mentioned a different type of bias (15\%). Some of them were even mistaken regarding evaluations of biases.

When they were asked about which parts of a CT are analysed before deciding to take part in a study, the specialists mentioned the following in order of priority:

- Characteristics of the population studied (93\%).

- Ethical considerations of the study (86\%).

- Representativeness of the study (64\%). 
Almost half of them admitted that they do not have sufficient ability to analyse a CT (43\%), and $50 \%$ of these recognised that they were lacking in statistical skills.

It was found that just $29 \%$ of the specialists analyse clinical protocols before prescribing new drugs in order to find out whether the population is similar to the one being treated or if the study was wellproduced methodologically; $64 \%$ do this sometimes.

Finally, some of them said that during the performance of a CT they had identified groups applying pressure for the use of a particular treatment $(T 37, T 38)$.

\section{Discussion}

More than $40 \%$ of the specialists who did not have a CT in progress in their hospital mentioned that the information about CT that they take as a basis is insufficient and they must be applied with modifications when they are to be used with the patients they are each treating, since the results of a CT cannot be applied to people who are outside the scope of the sample used ${ }^{3}$. There is clear concern among specialists who treat the elderly population in Spain, where life expectancy increased to 86 for women and 80 for men in $2016^{7}$. All of the specialists were of the opinion that real world data studies must be prioritised as a measure to be able to apply the proposed treatments to real patients.

The majority of interviewees who had a CT in their hospital did not receive financial benefits, in spite of these trials requiring special logistics and different levels of financing; it is important for the benefit always to be higher than the cost of carrying it out ${ }^{8}$.

The main interest of a CT is to respond to a patient's need, although sometimes CT are due to researchers' interests. This is important because CT must be useful in themselves, without the underlying interests mattering ${ }^{1}$; otherwise we would be moving away from patient-centred research ${ }^{9}$.

In order to achieve this, it is important for monitors to be adequately trained, since lack of training in professionals involved in clinical trials affects the development of the EC itself ${ }^{1}$, and it has been observed in this context that many doctors are underemployed as volunteers ${ }^{10}$.

In addition, the majority stated that sometimes it is the pharmaceutical companies that analyse the information from CT, and this creates suspicion among professionals. This fear could disappear if independent funding were found and there were no conflicts of interest with the pharmaceutical industry.

When performing a CT, one of the factors that may influence a patient's decision to participate is his/her socio-economic situation. This is in keeping with the results of other studies on adherence to treatment in which an association was identified between financial difficulties and a decrease in attendance by patients to receive treatment ${ }^{11}$. 
Moreover, one of the criteria for the specialist not including a patient in a CT is their lack of understanding, because the patient will not follow the experimental treatment or will do so irregularly. Another factor is the patient's complexity, the treatment's side-effects and the patient's lack of motivation ${ }^{3}$.

The specialists commented that they did not receive direct benefits for including a patient in research, at least no financial benefits, and only rarely academic benefits. The majority commented that they had not felt forced to include patients in a CT and recommended a CT convinced that it was the most appropriate therapeutic option. However, between 2014 and 2016, the Food and Drug Administration (FDA) in the United States approved 47 antineoplastics, only nine (19\%) of which complied with the standards of the American Society of Clinical Oncology to consider the drug as having a significant clinical benefit ${ }^{12}$.

$64.29 \%$ were of the opinion that it was very difficult to find an ideal patient to participate in a CT. In spite of this, many epidemiologists think that it is only through randomised controlled $\mathrm{CT}^{13-15}$ or metaanalyses $^{16,17}$ that reliable results can be proven, because it is believed that the more flexible the designs or analysis methods are, the less trustworthy the results are ${ }^{5}$.

Regarding the knowledge that the specialists have of CT, $51 \%$ of the interviewees mentioned that they were aware of selection bias. Although the risk of this bias appearing is intimately linked to clinical research ${ }^{18}$, few researchers are aware of the biases from which they may suffer. The most well-known selection biases are Berkson's or Neymann's bias, among others ${ }^{19,20}$, but there are other groups such as information, measurement, etc., which were not mentioned. Regarding this, one study showed that out of 704 articles, only 514 (73\%) had the help of an expert in methodology and statistics. The rejection rate was $71 \%$ for those that did not have these experts and $57 \%$ for those that $\operatorname{did}^{21}$.

It is concerning that $46 \%$ considered that they do not have sufficient ability to analyse a CT and that $50 \%$ recognised that they were lacking in statistical skills. There are those who are of the opinion that an oncologist should be knowledgeable in his/her field and not an expert in statistics. Others consider that any researcher knows enough about statistics ${ }^{3}$; this would explain some errors in the use of statistical evidence in $\mathrm{CT}^{22}$. Both forms of knowledge, clinical and statistical, must go hand-in-hand in a CT.

Some of the interviewees mentioned a kind of pressure from oncologists and pharmaceutical companies; regarding this, sponsored research initiatives should be patient-focused ${ }^{23}$ and professionals should accept the challenges of producing CT. Patient empowerment groups could help avoid the influence of biases in the future ${ }^{1}$. Patient associations can also exert pressure. One example is the case of interferon beta-1b, which was initially used to treat Multiple Sclerosis. It was approved without complying with the necessary specifications ${ }^{24}$ for safety and efficacy due to patient pressure. Another example is azidothymidine, which was also authorised due to pressure from patient associations and excess mortality ${ }^{25}$. The FDA implemented a fast track process to accelerate the approval of some protocols ${ }^{26}$. 


\section{Limitations}

Due to the small size of the population studied, a larger study will be required, with national coverage, in order to be able to generalise these results.

\section{Conclusions}

Decision-making concerning oncological treatments of therapies based on CT is hindered by the lack of correspondence between the patients included in the CT and patients seen in consultation. To make up for this deficiency, inclusion of real world data studies is proposed.

Aiding the professional progression of their research career is an incentive for clinicians to participate in a CT.

The need for training in critical reading and analysis of CT for this group has also been revealed.

One way of ending the distrust generated by CT performed by the pharmaceutical industry is for independent financial bodies to develop these kinds of studies.

\section{Declarations}

\section{Ethics approval and consent to participate}

Ethics

Ethics approval and consent to participate

Ethics approval

"Not applicable", because we haven't interviewed to patients, we ask the specialist opinion about different situations in relation with clinical trials, in a qualitative design research.

This manuscript does not report on or involve the use of any animal or human data or tissue.

Consent to participate

This study followed the recommendations of the Helsinki Declaration, all of the participants (Oncologist specialist's medical doctor) signed the informed consent and the necessary measures were taken to ensure anonymity.

Consent for publication

"Not applicable". This manuscript does not contain data from any individual person. 
Availability of data and materials

The datasets used and/or analysed during the current study are available from the corresponding author on reasonable request.

Competing interests

"The authors declare that we have no competing interests"

Funding

This document has been sponsored by Laboratorios Merck. The authors have not received any form of pressure or coercion in the design, analysis and final drafting of this document.

Authors' contributions

(AFM), (NML), (NML), (JCG), (EMBV), (SCF), (AAA), (DRC), (JCMM), they have contributed in study design

(ACC), (MCR), (EGM), (PGV), (BGS), (MJF), (MLPA), (AAA), (DRC),(JCMM),(RIGhT-Sens), they have contributed in analysis and interpretation of results.

(GQA), (CRR), (MSF), (FVR), (AAA), (DRC),(JCMM) they have written the discussion.

(DRC), (JCMM) they have reviewed the entire document and were the major contributors in writing of manuscript.

All authores read and approved the final manuscript.

All authors have contributed at each stage of the research process.

Acknowledgements

"Not applicable".

approval

"Not applicable", because we haven't interviewed to patients, we ask the specialist opinion about different situations in relation with clinical trials, in a qualitative design research.

\section{Consent for publication}

"Not applicable". This manuscript does not contain data from any individual person.

\section{Competing interests}


"The authors declare that we have no competing interests"

\section{Funding}

This document has been sponsored by Laboratorios Merck. The authors have not received any form of pressure or coercion in the design, analysis and final drafting of this document.

\section{Authors' contributions}

(AFM), (NML), (NML), (JCG), (EMBV), (SCF), (AAA), (DRC), (JCMM), they have contributed in study design

\section{Acknowledgements}

"Not applicable".

\section{References}

1. Ioannidis JPA. Why most clinical research is not useful. PLoS Med. 016;13(6):e1002049.

2. Macleod MR, Michie S, Roberts I, Dirnagl U, Chalmers I, loannidis JPA et al. Biomedical research: increasing value, reducing waste. Lancet. 2014;383(9912):101-4.

3. Silva Aycaguer LC. Frequent methodological errors in clinical research. Medicina Intensiva (English Edition). 2018;42(9):541-6.

4. Higgins JPT, Thomas J, Chandler J, Cumpston M, Li T, Page MJ et al., eds. Cochrane handbook for systematic reviews of interventions version 6.0 [internet]. Londres: Cochrane, 2019 [consultado 2 sep 2020]. Disponible en: www.training.cochrane.org/handbook.

5. Urrutia Egaña M, Barrios Araya S, Gutiérrez Núñez M, Mayorga Camus M. Métodos óptimos para determinar validez de contenido. Revista Cubana de Educación Médica Superior. 2014;28(3):547-58.

6. Gil-Gómez B, Pascual Ezama D. La metodología Delphi como técnica de estudio de validez de contenido. Anales de Psicología. 2012;28(3):1011-20.

7. Abellán García A, Ayala García A, Pérez Díaz J, Pujol Rodríguez R. Un perfil de las personas mayores en España, 2018. Indicadores estadísticos básicos. Informes Envejecimiento en Red. 2018;17:34.

8. Detsky AS. Are clinical trials a cost-effective investment? JAMA. 1989;262(13):1795-800.

9. Mullins CD, Vandigo J, Zheng Z, Wicks P. Patient-centeredness in the design of clinical trials. Value Health. 2014;17(4):471-5.

10. Emanuel EJ. Reinventing American health care. How the affordable care act will improve our terribly complex, blatantly unjust, outrageously expensive, grossly inefficient, error prone system. Nueva York: PublicAffairs; 2014. 
11. Culqui DR, Munayco CV, Grijalva CG, Cayla JA, Horna-Campos O, Alva CK et al. Factors associated with the non-completion of conventional anti-TB treatment in Peru. Arch Bronconeumol. 2012;48(5):150-5.

12. Kumar H, Fojo T, Mailankody S. Guidelines for oncology clinical trials. JAMA Oncol. 2016;2:1238-40.

13. Moher D, Schulz KF,Altman DG. The CONSORT statement: revised recommendations for improving the quality of reports of parallel-group randomised trials. Lancet. 2001;357:1191-4.

14. Ioannidis JPA, Evans SJ, Gotzsche PC, O’Neill RT, Altman DG, Schulz K et al. Better reporting of harms in randomized trials: an extension of the CONSORT statement. Ann Intern Med. 2004;141:7818.

15. International Conference on Harmonisation E9 Expert Working Group. ICH harmonised tripartite guideline. Statistical principles for clinical trials. Stat Med. 1999;18:1905-42.

16. Moher D, Cook DJ, Eastwood S, Olkin I, Rennie D, Stroup DF. Improving the quality of reports of metaanalyses of randomised controlled trials: the QUOROM statement. Quality of Reporting of Metaanalyses. Lancet. 1999;354:1896-900.

17. Stroup DF, Berlin JA, Morton SC, Olkin I, Williamson GD, Rennie D et al. Meta-analysis of observational studies in epidemiology: a proposal for reporting. Meta-analysis Of Observational Studies in Epidemiology (MOOSE) Group. JAMA. 2000;283(15):2008-12.

18. Manterola C, Otzen T. Los sesgos en investigación clínica. Int J Orphol. 2015;33(3):1156-64.

19. Lazcano-Ponce E, Fernández E, Salazar-Martínez E, Hernández-Ávila M. Estudios de cohorte. Metodología, sesgos y aplicación. Salud Pública Méx. 2000;42(3):230-41.

20. Manterola DC, Santander AC, Otzen HT. Cómo valorar e interpretar un artículo sobre pronóstico. Rev Chil Cir. 2013;65(1):77-84.

21. Altman PG, Goodman NS, Schroter S. How statistical expertise is used in medical research. JAMA. 2002;287:2817-20.

22. Greenland S, Senn SJ, Rothman KJ, Carlin JB, Poole C, Goodman SN et al. Statistical tests, $P$ values, confidence intervals, and power: a guide to misinterpretations. Eur J Epidemiol. 2016;31:337-50.

23. Fleurence RL, Curtis LH, Califf RM, Platt R, Selby JV, Brown JS. Launching PCORnet, a national patient-centered clinical research network. J Am Med Inform Assoc. 2014;21(4):578-82.

24. European Medicines Evaluation Agency. European public assessment report (EPAR) on betaferon (interferon beta-1b). CPMP/590/95.

25. Carné X, Costa J. Problemas y controversias en torno al EC. Barcelona: Fundación Dr. Antoni Esteve; 1998.

26. European Medicines Evaluation Agency. Accelerated evaluation of products indicated for serious diseases (life threatening or heavy disabling diseases). CPMP/495/96.

\section{Tables}

Due to technical limitations the Tables are available as downloads in the Supplemental Files. 


\section{Supplementary Files}

This is a list of supplementary files associated with this preprint. Click to download.

- Tabla1.xlsx

- Tabla2.xlsx

- Tabla3.xlsx

- Tabla4.xlsx 